\title{
Rational modes selection of magnetic treatment of metal surfaces
}

\author{
Oleg Burlachenko ${ }^{1}$, Alexander Lyashenko ${ }^{1, *}$, and Maxim Ivanov ${ }^{1}$ \\ ${ }^{1}$ Volgograd State Technical University, 40005Volgograd, Russian Federation
}

\begin{abstract}
The given article considers the problem of machine parts working capacity increase based on the complex surface treatment. The authors proposed perspective method of surface treatment that it should be in the elastically plastic deformation of friction surfaces in an alternating magnetic field. In addition, a mathematical apparatus, which makes it possible to reveal the most rational treatment regime, was proposed.
\end{abstract}

Ensuring the machines and mechanisms durability is one of the most important topics of modern scientific research in the field of engineering. This area is devoted to many scientific researches, which are based on various ways to change the surface layer of metals through all sorts of influences, in order to increase the contacting parts wear resistance.

The metals with increased wear resistance need in advanced development - space, military industry, etc. is particularly expressed [1-5]. That is why an in-depth analysis of existing methods, identification of the most highly effective ones among them and applying them with the greatest impact is an actual problem, which many well-known scientists concern in their researches in the field of engineering.

The apparatus for obtaining the wear resistance best indices in the various methods application is usually the mathematical modelling of rubbing surfaces triboengineering processes. Its application allows us to find reasonably the patterns of the various factors influence on the response function (wear resistance), and then predict its variation depending on the variation of the factors. Factor characteristics values search and change are carried out until the best results are obtained within the framework of the planned studies (Fig. 1).

As it is known [6-7], the task of a specific joint or unit wear resistance increasing does not often provide for a qualitative modification of the wear material structural composition in its full-blast, but takes into account only the modification of its surface layer, since the protection of the mating parts from wear is in some cases achieved by surface hardening. In the general case, surface strengthening is considered as an increase in the microhardness of a surface and other mechanical characteristics, such as roughness, elasticity, fracture toughness, etc. To improve the structural composition of the wear materials surface layer various methods of actuation are used: mechanical strengthening, surfacing, spraying, combined methods, etc[8-10]. This approach simplifies and reduces the cost of parts with increased performance production.

\footnotetext{
*Corresponding author: lyashenko2626@ mail.ru
} 


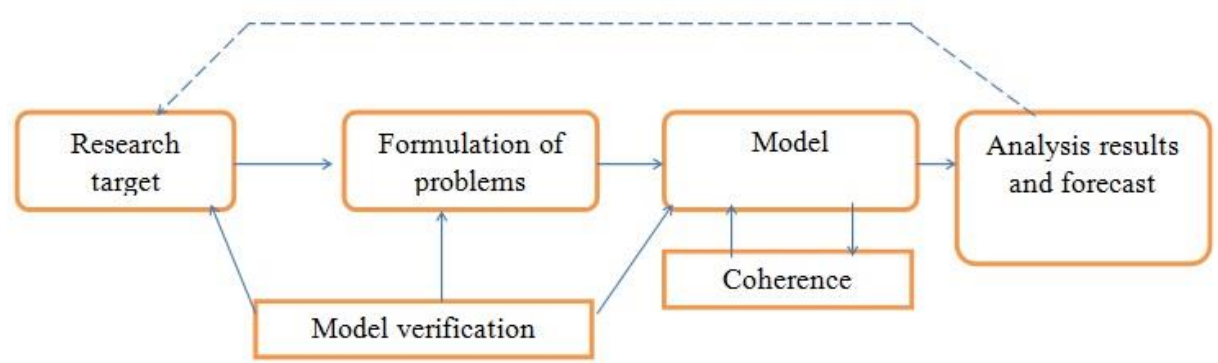

Fig. 1. Algorithm for mathematical model of triboengineering processes designing

One of the methods of increasing wear resistance, which is currently insufficiently studied and has a huge potential, is the use of metals magnetic treatment and combined methods based on it $[11,12]$. Selection of treatment processing modes varies depending on the particular installation, various factors of influence, processing method, etc. A huge number of factors combinations, the continuing study of the various kinds magnetic influences effects on metals and other materials - all this does not allow us to cover this area in full, therefore, the work to improve the properties of metals based on this treatment remains in demand today. Based on this, the combined magnetic treatment of metals was chosen as the "research target".

The principle of the chosen combined method consists in applying a surface plastic deformation in the alternating magnetic field. In this treatment method, the workpiece is placed in a closed space into which steel balls of a certain dimmer and a dopant are filled up. Due to the energy of the magnetic field, the steel balls and the alloying substance move in collision with the workpiece. In this case, the surface is deformed at the micro level - a more smooth and regular microrelief is formed, plasticity and microhardness are increased. At the same time, diffusion mass transfer to the surface layer of dopant molecules takes place, which is especially important in hard-to-work parts of the workpiece. It should also be noted that the diffusion mass transfer of the dopant onto the surface of the workpiece leads to the change in the physical, chemical, and electromagnetic characteristics of the friction surface, while the workpiece material itself does not change its original properties. This method, when selecting a dopant with the necessary properties, allows controlling the operational characteristics of the friction surfaces to be treated.

The selection of technological regimes for such a method of actuation on the metals surface layer consists in planning the experiment and in profound analysis of the results obtained.

To obtain maximum values of wear resistance with combined magnetic treatment, the concept of a sequential experiment was used as a basis for the research [13]. Among all possible approaches to the planning of the experiment, this method allows us to find with the least expenditure, gradually, in the first approximation the area of the factor space in which the maximum values of the desired function are found. The principle of the concept of a sequential experiment consists in carrying out random series of experiments in a given area and then moving towards obtaining maximum values, until the extremum of the sought response function is reached, provided that the selected factors are adequate.

Treatment period, the filling volume of the working chamber with pellet and the pellet diameter were chosen as factor characteristics (Table 1).

Since to search for the response function maximum values it is necessary to carry out a large number of expensive and labor-intensive experiments, it was decided not to conduct a full-factor experiment and fall back to a fractional-factor experiment. This is necessary in order to obtain in the studied process linear approximation first approximation and chosen 
by us factors influence on it. This approach proved to be more expedient in connection with the savings of funds and time in the search for maximum values.

Table 1. Factor characteristic.

\begin{tabular}{|c|c|c|c|}
\hline Factor characteristic & -1 & 0 & +1 \\
\hline Pellet diameter, $\mathrm{mm}\left(\mathrm{x}_{1}\right)$ & 2 & 3 & 4 \\
\hline Filling volume, $\%\left(\mathrm{x}_{2}\right)$ & 15 & 25 & 35 \\
\hline Treatment period, $\min .\left(\mathrm{x}_{3}\right)$ & 7 & 10 & 13 \\
\hline
\end{tabular}

Since to search for the response function maximum values it is necessary to carry out a large number of expensive and labor-intensive experiments, it was decided not to conduct a full-factor experiment and fall back to a fractional-factor experiment. This is necessary in order to obtain in the studied process linear approximation first approximation and chosen by us factors influence on it. This approach proved to be more expedient in connection with the savings of funds and time in the search for maximum values.

The most common method for designing a fractional-factor experiment plan is to search through the values of the variable factors only at the boundaries of their research (at the upper and lower levels) [14]. At one of the experimental points, three experiments were carried out, which is necessary to determine the experiment reproducibility dispersion.

The planning matrix (Table 2) was made according to the following rules:

1. Each g-th row of the matrix contains the set of coordinates $\mathrm{z}_{\mathrm{ig}}$ of the point at which the g-th experiment is performed $(\mathrm{i}=1,2, \ldots, \mathrm{n} ; \mathrm{g}=1,2, \ldots, \mathrm{N})$.

2. In the first line, all the controllable factors are selected at the lower level, i.e. $\mathrm{Zi}_{\mathrm{i}}=-1$. The subsequent g-th variants of variation in the plan design are chosen as follows: in the case of a line-byline search of all options, the frequency of alteration the change level of the controlled variables for each subsequent factor $\mathrm{z}_{\mathrm{i}}+1$ is half that of the previous one. Variation of controlled factors occurs on two levels -+1 and -1 .

3. Three experiments are added to the experiment plan at the main level (in plan, in theTable 3, these are the points of Plan No. 9.1 - 9.3).

Table 2. A shortened plan for the experiment of type $2^{3}$ carrying out.

\begin{tabular}{|c|c|c|c|c|}
\hline \multirow{2}{*}{$\begin{array}{l}\text { № of the } \\
\text { technological } \\
\text { regime }\end{array}$} & \multicolumn{3}{|c|}{ Factor characteristics. } & \multirow{2}{*}{$\begin{array}{l}\text { Effective characteristics. } \\
\mathrm{y}_{\mathrm{i}}\end{array}$} \\
\hline & $\mathrm{X}_{1}$ & $\mathrm{X}_{2}$ & $\mathrm{X}_{3}$ & \\
\hline 1 & +1 & +1 & +1 & $\mathrm{y}_{1}$ \\
\hline 2 & +1 & +1 & -1 & $\mathrm{y}_{2}$ \\
\hline 3 & +1 & -1 & +1 & $\mathrm{y}_{3}$ \\
\hline 4 & +1 & -1 & -1 & $\mathrm{y}_{4}$ \\
\hline 5 & -1 & +1 & +1 & $\mathrm{y}_{5}$ \\
\hline 6 & -1 & +1 & -1 & y6 \\
\hline 7 & -1 & -1 & +1 & $\mathrm{y}_{7}$ \\
\hline 8 & -1 & -1 & -1 & $\mathrm{y}_{8}$ \\
\hline 9.1 & 0 & 0 & 0 & y9.1 \\
\hline 9.2 & 0 & 0 & 0 & У9.2 \\
\hline 9.3 & 0 & 0 & 0 & У9.3 \\
\hline
\end{tabular}


Next, a series of experiments was carried out, in which factor characteristics were changed in accordance with the plan. The test samples had a steel grade of $40 \mathrm{X}$ with surface finish (roughness) $\mathrm{Ra}=1.25 \mathrm{~mm}$. According to the got data, it was necessary to obtain the regression equation by processing statistical data by mathematical methods.

Regression analysis consists of communication analytical form definition, in which the change in the effective characteristic is due to the influence of one or more factor characteristics, and the set of all other factors that also affect the effective characteristic is taken as constant and mean values [15]. In our case, the equation of the connection between the three variables $\mathrm{x}$ and the $\mathrm{y}$ response is as follows:

$$
y=\hat{f}\left(x_{1}, x_{2}, x_{3}\right)
$$

- where $y$ is the dependent variable (the resultant characteristic);

$-\mathrm{x}_{1}, \mathrm{x}_{2}, \mathrm{x}_{3}$ are independent explanatory variables (characteristic-factors).

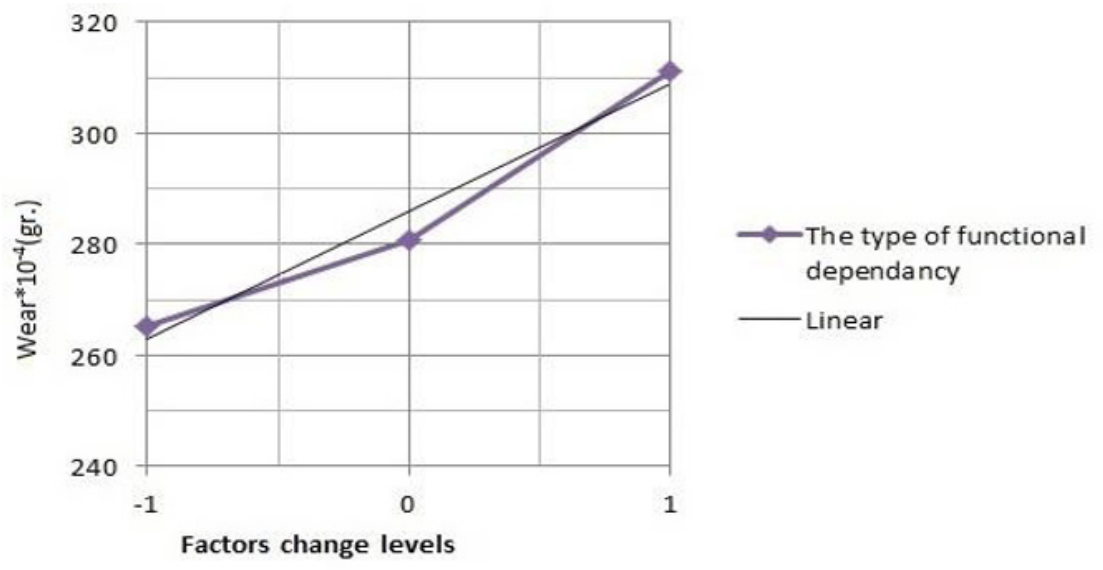

Fig. 2. General view of the change in wear resistance in the selected factor space.

As can be seen from the figure 2, to process the experimental data, it is expedient to choose the linear parabolic, or polynomial type of the regression equation.

To process the results of the experiment by the method of least squares, it was decided to use a linear equation:

$$
y=a_{0}+a_{1} x_{1}+a_{2} x_{2}+a_{3} x_{3}
$$

To calculate the coefficients of the equation using the method of least squares, a system of linear equations was composed, in general it has the following form: 


$$
\left\{\begin{array}{l}
a_{0} \cdot N+a_{1} \sum_{i=1}^{N} X_{1 i}+a_{2} \sum_{i=1}^{N} X_{2 i}+a_{3} \sum_{i=1}^{N} X_{3 i}=\sum_{i=1}^{N} y_{i} \\
a_{0} \sum_{i=1}^{N} X_{1 i}+a_{1} \sum_{i=1}^{N} X_{1 i}^{2}+a_{2} \sum_{i=1}^{N} X_{1 i} X_{2 i}+a_{3} \sum_{i=1}^{N} X_{1 i} X_{3 i}=\sum_{i=1}^{N} X_{1 i} y i \\
a_{0} \sum_{i=1}^{N} X_{2 i}+a_{1} \sum_{i=1}^{N} X_{2 i} X_{1 i}+a_{2} \sum_{i=1}^{N} X_{2 i}^{2}+a_{1} \sum_{i=1}^{N} X_{2 i} X_{3 i}=\sum_{i=1}^{N} X_{2 i} y_{i} \\
a_{0} \sum_{i=1}^{N} X_{3 i}+a_{1} \sum_{i=1}^{N} X_{3 i} X_{1 i}+a_{1} \sum_{i=1}^{N} X_{3 i} X_{2 i}+a_{2} \sum_{i=1}^{N} X_{3 i}^{2}=\sum_{i=1}^{N} X_{3 i} y_{i}
\end{array}\right.
$$

The solution of the equations system resulted in the following values of regression coefficients: $\mathrm{a}_{0}=0,035, \mathrm{a}_{1}=3,819, \mathrm{a}_{2}=0,059, \mathrm{a}_{3}=0,901$ form:

The regression equation after substitution of the obtained coefficients has the following

$$
y=0,035+3,819 x_{1}+0,059 x_{2}+0,901 x_{3}
$$

Having made the estimation of the obtained equation adequacy (Table №3 and Table №4), a conclusion was made about the obtained dependence adequacy.

\begin{tabular}{|c|c|c|c|c|c|}
\hline \multirow{3}{*}{$\begin{array}{c}\text { Type of the } \\
\text { equation }\end{array}$} & \multicolumn{5}{|c|}{ Value of t-criterion $(\mathrm{P}=0,95)$} \\
\hline & \multirow{2}{*}{$\begin{array}{c}\text { Tabular } \\
\text { value for } \\
\text { all the } \\
\text { coefficient } \\
\text { s }\end{array}$} & \multicolumn{4}{|c|}{ Calculable value } \\
\hline & & $\mathrm{a}_{0}$ & $\mathrm{a}_{1}$ & $\mathrm{a}_{2}$ & $\mathrm{a}_{3}$ \\
\hline Straight line & 2,31 & 2,01 & 1,29 & 2,21 & 1,1 \\
\hline
\end{tabular}

Table 3. Results of regression equations comparison.

\begin{tabular}{|c|c|c|c|}
\hline \multirow{2}{*}{$\begin{array}{c}\text { Type of the } \\
\text { equation }\end{array}$} & \multicolumn{2}{|c|}{ Value of F-criterion $(\mathrm{P}=0,95)$} & \multirow{2}{*}{$\varepsilon \%$} \\
\cline { 2 - 3 } & Calculable value & Tabular value & \\
\hline Straight line & 9,584 & 10,13 & 10 \\
\hline
\end{tabular}

Table 4. Results of testing the regression coefficients by the $t$-criterion.

Processing regression coefficients were determined as the result of the obtained experimental data, according to which it is possible to draw conclusions about the each combined treatment factors significance on the friction surfaces abrasion resistance. As can be seen from the obtained equation, the diameter of the fraction $\left(\mathrm{x}_{1}\right)$ and the processing period $\left(\mathrm{x}_{3}\right)$ are the factors that have the greatest impact on the wear resistance after combined magnetic treatment. By varying them and adding combined action other parameters to them, it is still possible to achieve the treated surface wear resistance increase by finding the optimum values of these factors, as well as attracting factors having a more significant influence on wear.

The wear value obtained from this empirical dependence has a discrepancy with theoretical data of no more than $10 \%$. This already indicates a sufficient level of the developed mathematical model, although the use of fractional-factor experiment leaves the prerequisites for more accurate researches in the zone of the factor space in which the maximum of the objective function (wear resistance) was obtained. Thus, this analytical apparatus makes it possible to determine the wear rate of the specimens' friction surfaces treated under different processing regimes, and thereby reveal the most rational treatment regime. In our case, this mode is №2 ( $\mathrm{x}_{1}=4 \mathrm{~mm}$., $\mathrm{x}_{2}=35 \%, \mathrm{x}_{3}=7 \mathrm{~min}$.), since in this mode of processing the minimum wear values were obtained from the entire series of experiments. 
Also, based on the obtained data (figure 3), it can be concluded that the use of the selected combined magnetic treatment is promising. Wear indicators decreased by an average of $20 \%$, which at low costs for this type of influence will make it possible to reduce the machines and mechanisms parts with high performance characteristics production cost.

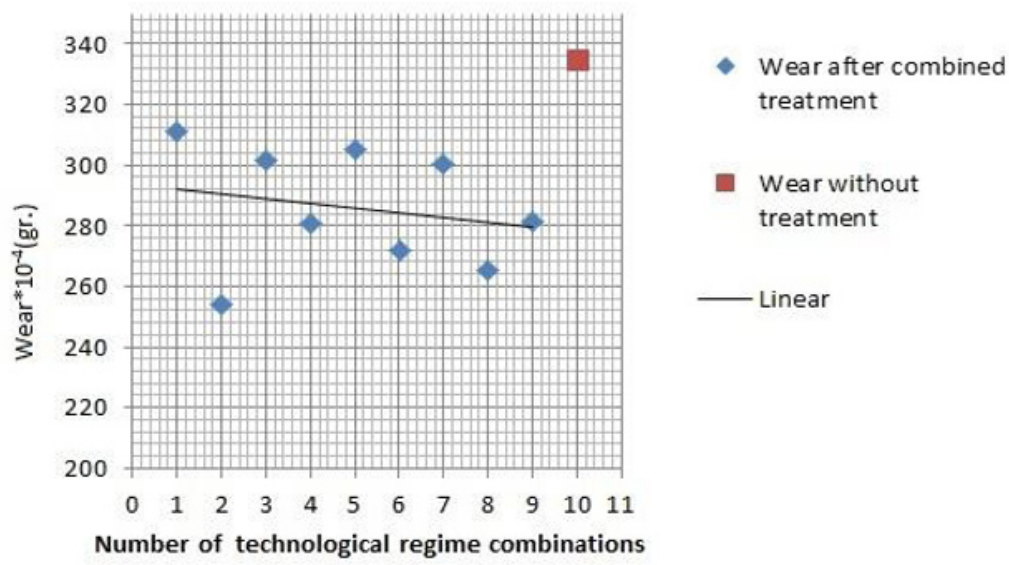

Fig. 3. Diagram of wear dependence on combined treatment technological modes

\section{References}

1. I. V. Kragelskii, Friction and Wear (Butterworths, London, 1965).

2. E.V. Kukushkin, V. A. Menovshchikov, Current issues of aviation and cosmonautics, pp.154-155, (2013).

3. N. D. Pronichev, P. A. Shulepov, Technological methods of ensuring the reliability of aircraft engines (Electronic resource,Samara, 2011.)

4. L.V. Fedorova, S.K. Fedorov, Yu.S. Ivanova, A.M. Lompas, News of higher educational institutions. Mech. eng., 9, pp. 85-92 (2017)

5. B.N. Pastuhov, Scientific problems of materials science (Moscow, 2009)

6. B. I. Kostetskii, I. G. Nosovskii, L. I. Bershadskiy, A. K. Karaulov, Reliability and durability of the machines (Techque, Kiev, 1975).

7. Ya.I. Shulyak, News of higher educational institutions. Mech. eng., 3, pp. 3-10 (2015)

8. O.V. Burlachenko, Yu.P. Serdobintsev, A.A. Lyashenko, MATEC Web Conf., 129, 1051 (2017).

9. O.V. Burlachenko, A.M. Burov, M.V. Ivanov, vestnik VolgGASU, 43, pp. 112-118 (2016)

10. O.V. Burlachenko, Technological support machine performance (VSUAE, Volgograd, 2002).

11. A.V. Komshina, A.S. Pomelnikova, Met. tech., 9, pp. 26-29 (2014)

12. Yu.V. Batigin, V. I. Lavinskiy, L.T. Himenko, Pulsed magnetic fields for advanced technologies.,1 (MOST-Tornado, Kharkov, 2003)

13. V.N. Kabanov, Yu.N. Nikolaev, Experimental study of the properties of concrete, mortar and dry mixes (VSUAE, Volgograd, 2015).

14. V.G. Galchenko, T.A. Gladkova, O.G. Berestneva, Planning and processing of experiment results (TPU, Tomsk, 2014)

15. V.E. Gmurman, Probability theory and mathematical statistics, 8 (Higher School, Moscow, 2002). 